\title{
Modelagem matemática da secagem da pimenta Cumari do Pará
}

\author{
Renata C. Reis ${ }^{1}$, Ludmilla S. Barbosa ${ }^{1}$, Mateus de L. Lima ${ }^{1}$, Jacqueline de S. Reis ${ }^{1}$, \\ Ivano A. D evilla² \& Diego P. R. Ascheri
}

\begin{abstract}
RESUMO
O bjetivou-se, neste trabalho, determinar e modelar as curvas de secagem da pimenta Cumari do Pará (Capsicum chinense Jacqui), nas temperaturas de 45,55 e $65 \stackrel{\circ}{\circ}$ e estimar seu coeficiente de difusão. As pimentas foram coletadas no município de Ceres, GO, e transportadas para UEG - Anápolis, GO; a secagem foi conduzida em secador experimental com controle de temperatura e fluxo de ar de $60 \mathrm{~m}^{3} \mathrm{~min}^{-1} \mathrm{~m}^{-2}$; os dados experimentais foram ajustados aos nove modelos matemáticos; as magnitudes do coeficiente de determinação $\left(R^{2}\right)$, do erro médio relativo $(P)$ e do erro médio estimado (SE) foram utilizadas para verificar o grau de ajuste dos modelos. Concluiu-se que: a) o comportamento das curvas de secagem da pimenta Cumari do Pará foi semelhante ao da maioria dos produtos agrícolas; b) o tempo de secagem foi de $4 \mathrm{~h} ; 2 \mathrm{~h}$ e 50 min e $1 \mathrm{~h}$ e 55 min para as temperaturas de secagem de 45 , 55 e $65^{\circ} \mathrm{C}$, respectivamente; c) o modelo matemático de secagem de Midilli et al. (2002), foi o que melhor se ajustou aos dados experimentais; d) os valores do coeficiente de difusão aumentaram de acordo com a el evação da temperatura do ar de secagem, apresentando resultados entre $2,2910^{-11}$ e $2,5710^{-11} \mathrm{~m}^{2} \mathrm{~s}^{-1}$.
\end{abstract}

Palavras-chave: modelos matemáticos, curvas de secagem, secador, Capsicum chinense Jacqui

\section{Mathematical modeling of drying kinetics of pepper Cumarí do Pará}

\begin{abstract}
Among the processes of food preservation, drying the product stands out for maintaining the nutritional quality and flavor of products. The objective of this study was to determine and adjust the curves of drying of Pará Cumari pepper (Capsicum chinense Jacqui), at temperatures of 45,55 and $65 \stackrel{\circ}{ } \mathrm{C}$. The Pará Cumari peppers were collected in the city of Ceres, GO and transported to UEG - A nápolis, GO. D rying was conducted in fixed bed dryer with temperature control and airflow of $60 \mathrm{~m}^{3} \mathrm{~min}^{-1} \mathrm{~m}^{-2}$. Experimental data were adjusted to nine mathematical models. The magnitude of the coefficient of determination $\left(R^{2}\right)$, the mean relative error $(\mathrm{P})$ and the estimated mean error (SE) were used to verify the degree of fit of the models. It was concluded that: a) the behavior of the drying curves of Pará Cumari pepper was similar to most agricultural products; b) the drying time was $4 \mathrm{~h} ; 2 \mathrm{~h}$ and 50 min and $1 \mathrm{~h}$ and $55 \mathrm{~min}$, respectively, for the drying temperatures of 45,55 and $65^{\circ} \mathrm{C}$; c) the mathematical model of drying proposed by Midilli et al. (2002) was the best fit to the experimental data; $d$ ) values of the diffusion coefficient increased with the increase of the temperature of the drying air, with results between $2.2910^{-11}$ and $2.5710^{-11} \mathrm{~m}^{2} \mathrm{~s}^{-1}$.
\end{abstract}

Key words: mathematical models, curves of drying, dryer, Capsicum chinense Jacqui

\footnotetext{
${ }^{1}$ Mestrando em Engenharia Agrícola da Universidade Estadual de Goiás - UEG, Br 153, n. 3.105, Fazenda Barreiro do Meio. CP 459. CEP 75132903. Anápolis, GO. Fone: (62) 9296-3430. E-mail: renataufg@gmail.com; agri milla@hotmail.com; jacque agroip@hotmail.com; mateusueg@yahoo.com.br

2 UEG. E-mail: devilla@ueg.br; ascheridpr@gmail.com
} 


\section{INTRODUÇÃO}

A importância das pimentas é atribuída às suas propriedades otimizadoras de sabor, aroma e cor, nos alimentos; os teores de vitamina $C$ total variam entre as espécies de pimenta, de 160 a

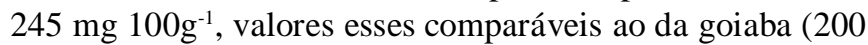
$\left.\mathrm{mg} 100 \mathrm{~g}^{-1}\right)$ e superiores ao da laranja $\left(60 \mathrm{mg} 100 \mathrm{~g}^{-1}\right)$. O mercado para as pimentas é muito segmentado e diversificado sendo dividido, basicamente, em produtos in natura, formas processadas e ornamentais (Gravina et al., 2004).

A espécie de pimenta Cumari do Pará (Capsicum chinense Jacqui) possui frutos de coloração amarela quando maduros, com cheiro forte e ardume ativo, medindo aproximadamente 2 a $3 \mathrm{~cm}$ de comprimento e $1 \mathrm{~cm}$ de largura sendo, por vezes, confundida com a Cumari verdadeira (EMATER, 2006).

O processo de secagem consiste na remoção de grande parte da água contida no produto a determinado nível, originando condições desfavoráveis à continuidade de suas atividades metabólicas e ao desenvolvimento de microorganismos (Martinazzo et al., 2007a).

Segundo Vilela \& Artur (2008) as informações contidas nas curvas de secagem são de fundamental importância para o desenvolvimento de processos e para o dimensionamento de equipamentos; com elas, pode-se estimar o tempo de secagem de certa quantidade de produtos e, com o tempo necessário para a produção, estima-se o gasto energético que refletirá no custo de processamento e, por sua vez, influenciará no preço final do produto. No dimensionamento de equipamento podese determinar as condições de operação para secagem e, com isto, a seleção de trocadores de calor, ventiladores e outros.

De acordo com Resende et al. (2008) as curvas de secagem em camada delgada variam com a espécie, variedade, condições ambientais, métodos de preparo pós-colheita, entre outros fatores; neste sentido, diversos modelos matemáticos têm sido utilizados para descrever o processo de secagem de produtos agrícolas.

O coeficiente de difusão $\left(\mathrm{D}_{\text {ef }}\right)$ é uma difusividade efetiva que engloba os efeitos de todos os fenômenos que podem intervir sobre a migração da água e seu valor é sempre obtido pelo ajuste de valores experimentais. Pode-se entender a difusividade como a facilidade com que a água é removida do material. Como a difusividade varia conforme mudam as condições de secagem (temperatura e velocidade do ar), ela não é intrínseca ao material; convenciona-se, então, chamá-la de difusividade efetiva (Oliveira et al., 2006).

Martinazzo et al. (2007b), secaram folhas de capim limão a $30,40,50$ e $60^{\circ} \mathrm{C}$ e obtiveram, como melhor modelo para estimar a secagem, o de Midilli et al. (2002). Os valores do coeficiente de difusão para esses valores de temperatura variaram de 4,00 $10^{-12}$ a 3,90 10 $10^{-11}$. Afonso Júnior \& Corrêa (1999), determinaram as curvas de secagem para sementes de feijão nas temperaturas de $35,40,45$ e $50{ }^{\circ} \mathrm{C}$ e verificaram que o modelo de Page foi o que melhor se ajustou aos dados de secagem; já Doymaz (2007), secou tomates em temperaturas de $55,60,65$ e $70{ }^{\circ} \mathrm{C}$, sobressaindo-se o modelo de Page com o melhor ajuste e os coeficientes de difusão variaram de 5,65 $10^{-10}$ a 7,53 $10^{-10} \mathrm{~m}^{2} \mathrm{~s}^{-1}$, conforme o aumento da temperatura.
De acordo com o exposto, este trabalho objetivou: a) determinar as curvas e o tempo de secagem da pimenta Cumari do Pará, nas temperaturas de 45,55 e $65^{\circ} \mathrm{C}$; b) ajustar modelos matemáticos de secagem aos dados experimentais obtidos na secagem da pimenta, definindo o melhor modelo ajustado; c) estimar o coeficiente de difusividade efetiva $\left(D_{\text {ef }}\right)$ e d) calcular a energia de ativação $\left(\mathrm{E}_{\mathrm{a}}\right)$ para a pimenta Cumari do Pará.

\section{Material E MÉTODOS}

As pimentas Cumari do Pará (Capsicum chinense Jacqui) foram coletadas no município de Ceres, GO, no mês de dezembro de 2009, e transportadas em temperatura ambiente em sacos plásticos, até a Unidade Universitária de Ciências Exatas e Tecnologias (UNUCET) da Universidade Estadual de Goiás (UEG), localizada na cidade de Anápolis, GO, onde foram armazenadas sob refrigeração até que a secagem fosse realizada.

Este estudo foi realizado no Laboratório de Secagem e Armazenamento de Produtos Vegetais, pertencente ao Curso de Engenharia Agrícola. As pimentas foram lavadas em água corrente e sanitizadas com solução de cloro a 200 ppm; em seguida, foram secadas a temperatura ambiente e cortadas transversalmente com faca em inox, adquirindo forma geométrica plana para a modelagem matemática e levadas às bandejas do secador. O teor de água do produto foi determinado pelo método padrão da estufa, a $105 \pm 3{ }^{\circ} \mathrm{C}$, por $24 \mathrm{~h}$, em três repetições (Brasil, 2009).

A secagem da pimenta Cumari do Pará foi conduzida em um secador experimental, com três bandejas circulares de fundo telado, conforme mostrado na Figura 1A e B. No estudo

A.

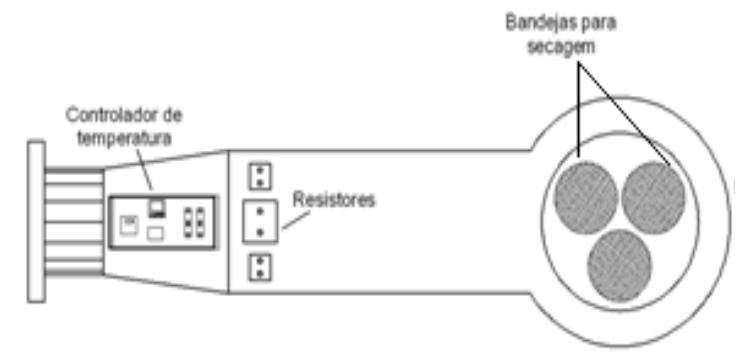

B.

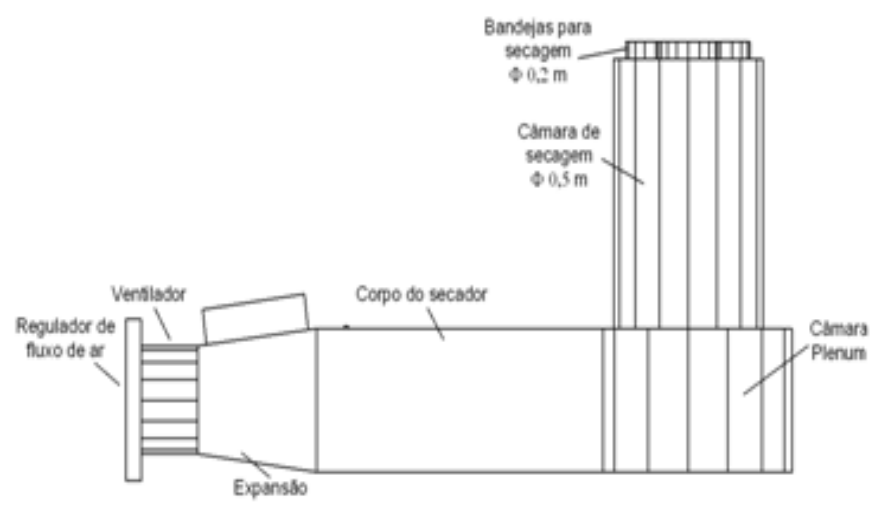

Figura 1. (A) vista superior do secador, (B) vista lateral do secador experimental 
utilizaram-se as temperaturas, para a secagem, de 45, 55 e $65^{\circ} \mathrm{C}$; o ar foi aquecido por um conjunto de resistências, totalizando $5 \mathrm{~kW}$, e o controle da temperatura foi feito por meio de um controlador tipo PID (N480 D) acoplado a uma das resistências.

Para o monitoramento da velocidade do ar de secagem na parte superior da bandeja, utilizou-se um anemômetro de pás rotativas e se manteve o ar com velocidade de $1 \mathrm{~m} \mathrm{~s}^{-1}$, o que equivale a $60 \mathrm{~m}^{3} \mathrm{~min}^{-1} \mathrm{~m}^{-2}$ de fluxo de ar; durante o processo de secagem as bandejas com as amostras foram pesadas periodicamente; a secagem da pimenta prosseguiu até que o produto atingisse seu teor de água de equilíbrio, este obtido por meio de três pesagens consecutivas, de forma a possibilitar o cálculo da razão de umidade do produto.

Aos dados experimentais foram ajustados os seguintes modelos matemáticos de secagem, Eqs. 1 a 9:

Modelo de Verna: RU = a exp $(-k \mathrm{t})+(1-\mathrm{a}) \exp \left(-\mathrm{k}_{1} \mathrm{t}\right)$

Modelo Logaritmico: $\mathrm{RU}=\mathrm{a} \exp (-\mathrm{k} \mathrm{t})+\mathrm{c}$

Modelo de Midilli et al.: $\mathrm{RU}=\mathrm{a} \exp \left(-\mathrm{k} \mathrm{t}^{\mathrm{n}}\right)+\mathrm{bt}$

Modelo de Page: $\mathrm{RU}=\exp \left(-\mathrm{k} \mathrm{t}^{\mathrm{n}}\right)$

Modelo de Newton: RU $=\exp (-\mathrm{k} \mathrm{t})$

Modelo de Henderson e Pabis: RU $=\mathrm{a} \exp (-\mathrm{k} \mathrm{t})$

Modelo de H. \& P. modificado: $\mathrm{RU}=\mathrm{a} \exp (-\mathrm{k} \mathrm{t})+$

$+\mathrm{b} \exp \left(-\mathrm{k}_{0} \mathrm{t}\right)+\mathrm{c} \exp \left(-\mathrm{k}_{1} \mathrm{t}\right)$

Modelo de Dois termos: RU $=\mathrm{a} \exp \left(-\mathrm{k}_{0} \mathrm{t}\right)+\mathrm{b} \exp \left(-\mathrm{k}_{1} \mathrm{t}\right)(8)$

Modelo de Aproximação da difusão: $\mathrm{RU}=\mathrm{a} \exp (-\mathrm{k} \mathrm{t})+$

$+(1-a) \exp (-k b t)$

em que:

RU - razão de umidade do produto, adimensional

$\mathrm{t}$ - tempo de secagem, $\mathrm{h}$

$\mathrm{k}, \mathrm{k}_{0}, \mathrm{k}_{1}$ - coeficientes de secagem, $\mathrm{s}^{-1}$

$\mathrm{a}, \mathrm{b}, \mathrm{c}, \mathrm{n}$ - constantes dos modelos, adimensional

Para o cálculo da razão de umidade (RU) durante as secagens nas diferentes temperaturas, utilizou-se a expressão:

$$
\mathrm{RU}=\frac{\mathrm{U}-\mathrm{U}_{\mathrm{e}}}{\mathrm{U}_{\mathrm{i}}-\mathrm{U}_{\mathrm{e}}}
$$

em que:

U - teor de água do produto, decimal b.s.

$\mathrm{U}_{\mathrm{i}}$ - teor de água inicial do produto, decimal b.s.

$\mathrm{U}_{\mathrm{e}}$ - teor de água de equilíbrio do produto, decimal b.s.

As magnitudes do coeficiente de determinação $\left(\mathrm{R}^{2}\right)$, do erro médio relativo $(\mathrm{P})$ e do erro médio estimado $(\mathrm{SE})$ foram utilizadas para verificar o grau de ajuste dos modelos, que foram calculados conforme as Eqs. 11 e 12:

$$
\begin{gathered}
\mathrm{P}=\frac{100}{\mathrm{n}} \sum \frac{\left|\mathrm{Y}-\mathrm{Y}_{0}\right|}{\mathrm{Y}} \\
\mathrm{SE}=\sqrt{\frac{\sum\left(\mathrm{Y}-\mathrm{Y}_{0}\right)^{2}}{\mathrm{GLR}}}
\end{gathered}
$$

em que:

$$
\begin{aligned}
& \mathrm{Y} \text { - valor observado experimentalmente } \\
& \mathrm{Y}_{0} \text { - valor calculado pelo modelo } \\
& \mathrm{n} \text { - número de observações experimentais } \\
& \text { GLR - graus de liberdade do modelo }
\end{aligned}
$$

O coeficiente de difusão efetivo foi obtido por meio do ajuste do modelo matemático da difusão líquida, descrito pela Eq. 13, aos dados experimentais da secagem da pimenta. Esta equação é a solução analítica para a segunda lei de Fick, considerandose a forma geométrica do produto como aproximação a uma placa plana, uma vez que a pimenta foi cortada ao meio, desconsiderando-se a contração volumétrica da mesma (Brooker et al., 1992).

$$
\begin{aligned}
R U= & \frac{U-U_{e}}{U_{i}-U_{e}}=\frac{8}{\pi^{2}} \sum_{n=0}^{\infty} \frac{1}{(2 n+1)^{2}} \exp - \\
& -\left[\frac{(2 n+1)^{2} \pi^{2} D_{e f} t}{4}\left(\frac{S}{V}\right)^{2}\right]
\end{aligned}
$$

em que:

$\mathrm{D}_{\text {ef }}$ - coeficiente de difusão efetivo, $\mathrm{m}^{2} \mathrm{~s}^{-1}$

$\mathrm{S}$ - área da placa equivalente, $\mathrm{m}^{2}$

$\mathrm{V}$ - volume da placa equivalente, $\mathrm{m}^{3}$

$\mathrm{t}$ - tempo, $\mathrm{h}$

n - número de termos

A solução analítica dessa equação apresenta-se na forma de uma série infinita e, portanto, o número finito de termos (n) no truncamento poderá determinar a precisão dos resultados.

Para avaliar a influência da temperatura no coeficiente de difusão efetivo, utilizou-se a equação de Arrhenius, descrita na Eq. 14:

$$
\mathrm{D}_{\text {ef }}=\mathrm{D}_{0} \exp \left(\frac{\mathrm{E}_{\mathrm{a}}}{\mathrm{RT}_{\mathrm{a}}}\right)
$$

em que:

$\mathrm{D}_{\mathrm{o}}$ - fator pré-exponencial, $\mathrm{m}^{2} \mathrm{~s}^{-1}$

$\mathrm{E}_{\mathrm{a}}$ - energia de ativação, $\mathrm{kJ} \mathrm{mol}^{-1}$

$\mathrm{R}$ - constante universal dos gases, $8,314 \mathrm{~kJ} \mathrm{kmol}^{-1} \mathrm{~K}^{-1}$

$\mathrm{T}_{\mathrm{a}}$ - temperatura absoluta, $\mathrm{K}$

\section{RESULTADOS E DISCUSSÃO}

O teor de água inicial para a pimenta Cumari do Pará foi de $82,69 \%$ b.s; na Figura 2 se apresenta a curva de secagem nas temperaturas de 45,55 e $65^{\circ} \mathrm{C}$, para esta pimenta.

Nota-se que, com o aumento da temperatura do ar de secagem, ocorre maior taxa de remoção de água do produto evidenciando o aumento da taxa e diminuição do tempo de secagem, concordando com os inúmeros resultados relatados 


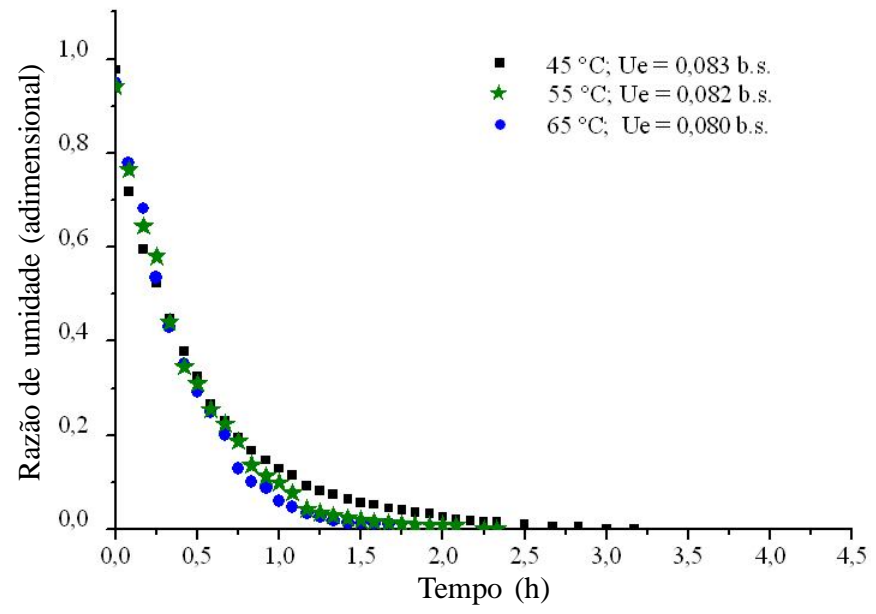

Figura 2. Curvas de secagem da pimenta Cumari do Pará (Capsicum chinense Jacqui) nas temperaturas de 45, 55 e $65^{\circ} \mathrm{C}$; U midade de Equilíbrio ( $\mathrm{U}_{\mathrm{e}}$ ) em base seca (b.s.)

por pesquisadores (Lahsasni et al., 2004; Babalis \& Belessiotis, 2004; Mohapatra \& Rao, 2005). Para as temperaturas de 45, 55 e $65^{\circ} \mathrm{C}$, utilizaram-se tempos de $4 \mathrm{~h} ; 2 \mathrm{~h}$ e 50 min e $1 \mathrm{~h}$ e $55 \mathrm{~min}$, respectivamente, para que a pimenta atingisse a umidade de equilíbrio.

$\mathrm{Na}$ Tabela 1 se encontram os parâmetros estatísticos utilizados para a comparação entre os nove modelos de secagem analisados, nas diversas condições de secagem utilizadas para a pimenta.

Para as três temperaturas utilizadas na secagem da pimenta verifica-se que todos os modelos matemáticos ajustados aos dados experimentais, apresentaram coeficientes de determinação $\left(\mathrm{R}^{2}\right)$ superiores a 0,98 , sendo o modelo de Midilli et al. (2002) (Eq. 3) o que possui maior valor de $\mathrm{R}^{2} \mathrm{em}$ todas as temperaturas de secagem estudadas. De acordo com Madamba et al. (1996), o coeficiente de determinação $\left(\mathrm{R}^{2}\right)$, não constitui, sozinho, um bom critério para a seleção de modelos não lineares; para isto, os valores para o erro médio estimado (SE) e erro médio relativo $(\mathrm{P})$ foram considerados. Os valores de $\mathrm{P}$ indicam o desvio dos valores observados em relação à curva estimada pelo modelo (Kashani-Nejad et al., 2007) e, segundo Mohapatra \& Rao (2005), valores inferiores a 10\% são recomendados para a seleção de modelos.

Analisando o SE nota-se que, para as temperaturas em estudo, o modelo de Midilli et al. obteve os menores valores, seguido dos modelos de aproximação por difusão (Eq. 9) e Henderson e Pabbis Modificado (Eq. 7). Para o valor do erro médio relativo (P) o de Midilli et al. foi o mais satisfatório, seguido também pelos modelos de aproximação por difusão e Henderson e Pabbis Modificado.

Desta forma, os melhores modelos para descrição da secagem da pimenta Cumarí do Pará são os de Midilli et al. (2002), aproximação por difusão e Henderson e Pabbis Modificado; assim, nas condições em que este trabalho foi realizado o modelo de Midilli et al. (2002), foi selecionado para representar a cinética de secagem da pimenta Cumari do Pará.

A Figura 3 apresenta o bom ajuste do modelo de Midilli et al. (2002) na descrição dos resultados experimentais e calculados da razão de umidade em função do tempo de secagem.

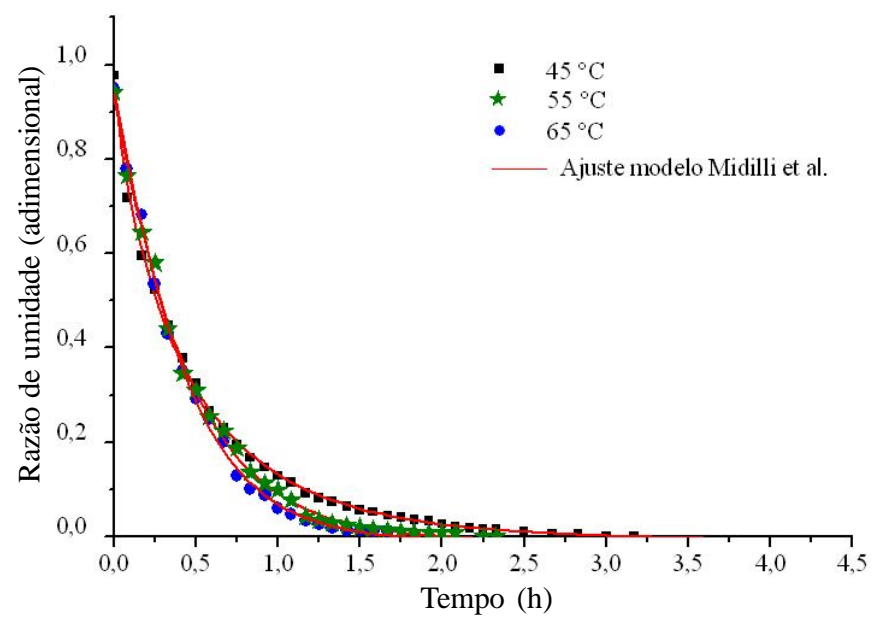

Figura 3. Valores reais e estimados de razão de umidade pela estimativa dos parâmetros do modelo de Midilli et al. (2002) nas diferentes temperaturas de secagem

Os valores dos parâmetros obtidos em cada modelo estão apresentados na Tabela 2, para as diferentes condições experimentais.

Para o modelo de Midilli et al. (2002), é possível observar que tanto o coeficiente de secagem $\mathrm{k}$ quanto a constante $\mathrm{n}$ aumentaram com o aumento da temperatura; já a constante b diminuiu com o aumento da temperatura e a constante a não demonstrou comportamento em função da temperatura de secagem. Martinazzo et al. (2007b) encontraram, como melhor

Tabela 1. Coeficiente de determinação $\left(R^{2}\right)$ erro estimado (SE) e erro médio relativo (P), calculados para verificação do ajuste dos model os matemáticos aos valores experimentais da secagem da pimenta Capsicum chinense Jacqui, obtidos nas temperaturas de 45,55 e $65^{\circ} \mathrm{C}$

\begin{tabular}{|c|c|c|c|c|c|c|c|c|c|}
\hline \multirow{2}{*}{$\begin{array}{c}\text { Modelos } \\
\text { matemáticos }\end{array}$} & \multicolumn{3}{|c|}{$\mathbf{R}^{2}$ (decimal) } & \multicolumn{3}{|c|}{ SE (decimal) } & \multicolumn{3}{|c|}{$\mathbf{P}(\%)$} \\
\hline & $45^{\circ} \mathrm{C}$ & $55^{\circ} \mathrm{C}$ & $65^{\circ} \mathrm{C}$ & $45^{\circ} \mathrm{C}$ & $55^{\circ} \mathrm{C}$ & $65^{\circ} \mathrm{C}$ & $45^{\circ} \mathrm{C}$ & $55^{\circ} \mathrm{C}$ & $65^{\circ} \mathrm{C}$ \\
\hline Verna & 0,991 & 0,993 & 0,996 & 0,018 & 0,013 & 0,011 & 10,957 & 5,683 & 4,351 \\
\hline Logarítmico & 0,997 & 0,997 & 0,997 & 0,010 & 0,008 & 0,008 & 49,180 & 9,390 & 7,671 \\
\hline Midilli et al. & 0,999 & 0,997 & 0,998 & 0,004 & 0,008 & 0,007 & 6,807 & 7,324 & 4,524 \\
\hline Page & 0,998 & 0,994 & 0,995 & 0,006 & 0,012 & 0,011 & 19,655 & 8,186 & 6,054 \\
\hline Newton & 0,983 & 0,993 & 0,995 & 0,018 & 0,012 & 0,012 & 10,957 & 6,413 & 9,128 \\
\hline Hend. e Pabis & 0,996 & 0,997 & 0,996 & 0,010 & 0,009 & 0,012 & 7,710 & 8,600 & 10,643 \\
\hline Hend. e P. Modif & 0,996 & 0,997 & 0,996 & 0,009 & 0,008 & 0,011 & 7,709 & 8,600 & 10,443 \\
\hline Dois termos & 0,996 & 0,997 & 0,996 & 0,010 & 0,008 & 0,013 & 7,719 & 8,600 & 10,643 \\
\hline Aprox. da Difusão & 0,998 & 0,993 & 0,995 & 0,004 & 0,012 & 0,012 & 6,943 & 6,248 & 9,128 \\
\hline
\end{tabular}


Tabela 2. Parâmetros obtidos dos modelos ajustados aos dados de secagem da pimenta Capsicum chinense Jacqui

\begin{tabular}{|c|c|c|c|c|c|c|c|}
\hline Modelos matemáticos & & & & arâmetro & & & \\
\hline Verna & $\begin{array}{c}\mathrm{T}\left({ }^{\circ} \mathrm{C}\right) \\
45 \\
55 \\
65\end{array}$ & $\begin{array}{c}a \\
-7,704 \\
-4,677 \\
-3,019\end{array}$ & $\begin{array}{c}k \\
2,284 \\
2,139 \\
1,773\end{array}$ & $\begin{array}{c}\mathrm{k}_{1} \\
2,284 \\
2,184 \\
1,934\end{array}$ & & & \\
\hline Logarítmico & $\begin{array}{l}45 \\
55 \\
65 \\
\end{array}$ & $\begin{array}{c}a \\
0,898 \\
0,952 \\
0,988 \\
\end{array}$ & $\begin{array}{c}k \\
2,165 \\
2,181 \\
2,276 \\
\end{array}$ & $\begin{array}{c}c \\
0,014 \\
-0,014 \\
-0,028 \\
\end{array}$ & & & \\
\hline Midilli et al. & $\begin{array}{l}45 \\
55 \\
65\end{array}$ & $\begin{array}{c}a \\
0,967 \\
0,930 \\
0,940\end{array}$ & $\begin{array}{c}\mathrm{k} \\
1,977 \\
2,283 \\
2,546\end{array}$ & $\begin{array}{c}n \\
0,812 \\
1,045 \\
1,107\end{array}$ & $\begin{array}{c}b \\
-0,002 \\
-0,004 \\
-0,005\end{array}$ & & \\
\hline Page & $\begin{array}{l}45 \\
55 \\
65\end{array}$ & $\begin{array}{c}k \\
2,039 \\
2,373 \\
2,624\end{array}$ & $\begin{array}{c}n \\
0,796 \\
0,970 \\
1,040 \\
\end{array}$ & & & & \\
\hline Newton & $\begin{array}{l}45 \\
55 \\
65\end{array}$ & $\begin{array}{c}\mathrm{k} \\
2,284 \\
2,373 \\
2,624 \\
\end{array}$ & & & & & \\
\hline Henderson e Pabis & $\begin{array}{l}45 \\
55 \\
65 \\
\end{array}$ & $\begin{array}{c}a \\
0,901 \\
0,946 \\
0,972 \\
\end{array}$ & $\begin{array}{c}k \\
2,040 \\
2,289 \\
2,484 \\
\end{array}$ & & & & \\
\hline Henderson e Pabis Modificado & $\begin{array}{l}45 \\
55 \\
65 \\
\end{array}$ & $\begin{array}{c}a \\
0,300 \\
0,315 \\
0,324 \\
\end{array}$ & $\begin{array}{c}k \\
2,040 \\
2,289 \\
2,484 \\
\end{array}$ & $\begin{array}{c}b \\
0,300 \\
0,315 \\
0,324 \\
\end{array}$ & $\begin{array}{c}\mathrm{k}_{0} \\
2,040 \\
2,289 \\
2,484 \\
\end{array}$ & $\begin{array}{c}C \\
0,300 \\
0,315 \\
0,324 \\
\end{array}$ & $\begin{array}{c}\mathrm{k}_{1} \\
2,040 \\
2,289 \\
2,484 \\
\end{array}$ \\
\hline Dois termos & $\begin{array}{l}45 \\
55 \\
65 \\
\end{array}$ & $\begin{array}{c}a \\
0,450 \\
0,473 \\
0,486 \\
\end{array}$ & $\begin{array}{c}k_{0} \\
2,040 \\
2,289 \\
2,484 \\
\end{array}$ & $\begin{array}{c}b \\
0,450 \\
0,473 \\
0,486 \\
\end{array}$ & $\begin{array}{c}k_{1} \\
2,040 \\
2,289 \\
2,484 \\
\end{array}$ & & \\
\hline Aproximação da Difusão & $\begin{array}{l}45 \\
55 \\
65\end{array}$ & $\begin{array}{c}a \\
0,812 \\
0,776 \\
0,776\end{array}$ & $\begin{array}{c}k \\
1,840 \\
2,552 \\
2,552\end{array}$ & $\begin{array}{c}b \\
14,165 \\
15,738 \\
16,445\end{array}$ & & & \\
\hline
\end{tabular}

modelo para a secagem de folhas de capim limão, o de Midilli et al. (2002), o que também teve sua forma geométrica considerada como placa plana e perceberam que o coeficiente de secagem $\mathrm{k}$ e a constante $\mathrm{n}$ aumentaram com o aumento da temperatura, confirmando os dados obtidos neste trabalho.

Resultado semelhante foi constatado por Radünz et al. (2010), que secaram folhas de sálvia com temperatura entre 40 e $90{ }^{\circ} \mathrm{C}$ e concluíram que os modelos de Handerson e Pabis e Midilli et al. (2002), foram os que representaram a melhor cinética de secagem para as folhas de sálvia.

Com base nos valores apresentados na Tabela 2, realizou se ajuste de equações para determinação dos valores dos parâmetros do modelo de Midilli et al. (2002), em função da temperatura do ar de secagem (Tabela 3 ).

Tabela 3. Parâmetros do modelo de Midilli et al. (2002) para cálculo da razão de umidade (RU ) da pimenta, em função da temperatura do ar de secagem

\begin{tabular}{|c|c|c|c|c|}
\hline \multirow{2}{*}{ Parâmetro } & \multirow{2}{*}{ Equação } & $\mathbf{R}^{2}$ & SE & \multirow{2}{*}{$\begin{array}{c}P \\
(\%)\end{array}$} \\
\hline & & \multicolumn{2}{|c|}{ (decimal) } & \\
\hline Valor médio: & \multicolumn{4}{|c|}{$a=0,946$} \\
\hline$k$ & $k=0,704+0,028 T$ & 0,999 & 0,016 & 2,875 \\
\hline$n$ & $\mathrm{n}=0,177+0,015 T$ & 0,948 & 0,070 & 2,066 \\
\hline$b$ & $b=0,004-0,002 T$ & 0,981 & 0,040 & 1,121 \\
\hline
\end{tabular}

Desta forma, os parâmetros $\mathrm{k}, \mathrm{n}$ e b, foram ajustados à equação linear, obtendo-se coeficientes de determinação $\left(\mathrm{R}^{2}\right)$ maiores que 0,94 , valores de erro médio relativo $(\mathrm{P})$ menores que 2,875 e valores de erro médio estimado (SE) menores que 0,070 .

Em virtude do mecanismo de transporte da água no interior do produto ocorrer por meio de difusão de vapor, utilizou-se a Eq. 13, baseada na Lei de Fick, com oito termos para produtos com forma geométrica de placa plana, para calcular, a partir dos dados experimentais, a difusividade efetiva do produto $\left(\mathrm{D}_{\mathrm{ef}}\right)$. Os valores encontrados para cada condição de secagem estão descritos na Tabela 4.

Nota-se que os valores da difusividade aumentaram com a elevação da temperatura de secagem, variando de 2,29 $10^{-11}$ a

Tabela 4. Valores da difusividade efetiva $\left(D_{\text {ef }}\right)$ obtidos para pimenta Cumari do Pará em diferentes temperaturas do ar de secagem

\begin{tabular}{ccccc}
\hline $\begin{array}{c}\text { Temperatura } \\
\left({ }^{\circ} \mathbf{C}\right)\end{array}$ & $\begin{array}{c}\mathbf{D}_{\text {ef }} \\
\left(\mathbf{1 0} \mathbf{~}^{-11} \mathbf{~}^{-\mathbf{1}}\right)\end{array}$ & $\begin{array}{c}\mathbf{R}^{\mathbf{2}} \\
(\%)\end{array}$ & $\begin{array}{c}\mathbf{S E} \\
\text { (adimensional) }\end{array}$ & $\begin{array}{c}\mathbf{P} \\
(\%)\end{array}$ \\
45 & 2,291 & 0,999 & 0,008 & 6,000 \\
55 & 2,393 & 0,976 & 0,057 & 4,219 \\
65 & 2,570 & 0,973 & 0,032 & 1,016 \\
\hline
\end{tabular}


2,57 $10^{-11} \mathrm{~m}^{2} \mathrm{~s}^{-1}$ para as temperaturas de 45 e $65{ }^{\circ} \mathrm{C}$, respectivamente. Segundo Zogzas et al. (1996), os valores de $\mathrm{D}_{\text {ef }}$ para produtos alimentícios se encontram na faixa de $10^{-11} \mathrm{a}$ $10^{-9} \mathrm{~m}^{2} \mathrm{~s}^{-1}$; para Rizvi (1995), o coeficiente de difusão efetivo é dependente da temperatura do ar de secagem, além da variedade e composição dos materiais, dentre outros. Almeida et al. (2009), secaram grãos de feijão da variedade Adzuki na faixa de temperatura de 30 a $70^{\circ} \mathrm{C}$ e obtiveram coeficientes de difusão na faixa de $0,5110^{-10}$ a $2,2310^{-10} \mathrm{~m}^{2} \mathrm{~s}^{-1}$, sendo o menor valor para a temperatura de $30{ }^{\circ} \mathrm{C}$ e o maior valor para a temperatura de 70 ${ }^{\circ} \mathrm{C}$. Amendola \& Queiroz (2007) encontraram ao secar banana a $50{ }^{\circ} \mathrm{C}$, valor de $1,65 \times 10^{-6} \mathrm{~m}^{2} \mathrm{~h}^{-1}$ para o coeficiente de difusão, $\mathrm{o}$ que corresponde a $4,5810^{-10} \mathrm{~m}^{2} \mathrm{~s}^{-1}$; logo, os valores do presente trabalho foram inferiores aos encontrados para o feijão e banana.

A Figura 4 mostra os valores de $\mathrm{D}_{\text {ef }}$ apresentados na forma de "In $\mathrm{D}_{\mathrm{e}}$ " descritos em função do recíproco da temperatura absoluta $\left(\mathrm{T}^{-1}\right)$; o ajuste linear obtido indica que houve uniformidade de variação da difusividade com a temperatura, sendo o valor da variação do coeficiente de difusividade obtido a $65^{\circ} \mathrm{C}$, um pouco superior em relação às temperaturas de $45 \mathrm{e}$ $55^{\circ} \mathrm{C}$, fato explicado em razão da vibração molecular de água pois, segundo Goneli (2007), a variação do coeficiente de difusão efetivo se dá com a elevação da temperatura, o que aumenta o nível de vibração molecular das moléculas de água e contribui para uma difusão mais rápida. Pode-se dizer, portanto, que houve uma difusão maior na temperatura de 65 ${ }^{\circ} \mathrm{C}$.

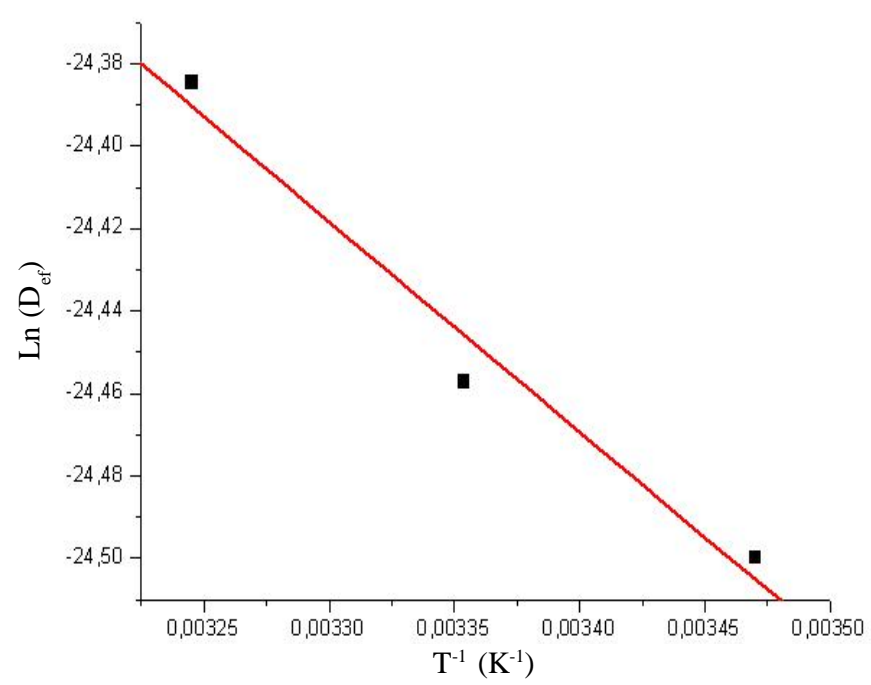

Figura 4. Representação de Arrhenius para a relação entre a difusi vidade efetiva ( $D_{\text {ef }}$ ) e a temperatura absoluta na secagem da Pimenta Cumari do Pará

A inclinação da curva da representação de Arrhenius fornece a relação $E a \mathrm{R}^{-1}$ enquanto a interseção com o eixo das ordenadas indica o valor de Do; a Eq. 15 apresenta os coeficientes da equação ajustada para o coeficiente de difusão efetivo da pimenta Cumari do Pará, calculados de acordo com a Eq. 14.

$$
D_{\text {ef }}=-6,278 \times 10^{-7} \exp \left(\frac{-5020,490}{R T}\right)
$$

A energia de ativação $\left(\mathrm{E}_{\mathrm{a}}\right)$ calculada da reta obtida na Figura 4, foi de $5,02 \mathrm{~kJ} \mathrm{~mol}^{-1}$. Nos processos de secagem, quanto menor a energia de ativação maior será a difusividade de água no produto (Goneli, 2007). Segundo Kashani-Nejad et al. (2007) a energia de ativação é uma barreira que deve ser ultrapassada para que o processo de difusão possa ser desencadeado no produto.

\section{CONCLUSÕES}

1. O comportamento das curvas de secagem da pimenta Cumari do Pará é o mesmo que os obtidos para a maioria dos produtos agrícolas e o tempo de secagem foi de $4 \mathrm{~h} ; 2 \mathrm{~h}$ e 50 min e 1 h e $55 \mathrm{~min}$, respectivamente, para as temperaturas de secagens de 45,55 e $65^{\circ} \mathrm{C}$;

2. O modelo matemático de secagem proposto por Midilli et al. (2002) foi o que melhor se ajustou aos dados experimentais;

3. Os valores do coeficiente de difusão aumentaram de acordo com a elevação da temperatura do ar de secagem, apresentando resultados entre 2,29 $10^{-11}$ a 2,57 $10^{-11} \mathrm{~m}^{2} \mathrm{~s}^{-1}$;

4. A dependência da difusividade em relação à temperatura foi descrita pela equação de Arrhenius, por meio da qual se obteve o valor da energia de ativação de $5,02 \mathrm{~kJ} \mathrm{~mol}^{-1}$.

\section{AgradeCimentos}

Os autores agradecem à CAPES, pelo auxilio financeiro concedido por meio de bolsa, aos mestrandos do curso de Engenharia Agrícola da Universidade Estadual de Goiás.

\section{LITERATURA CITADA}

Afonso Júnior, P. C.; Corrêa, P. C. Comparação de modelos matemáticos para descrição da cinética de secagem em camada fina de sementes de feijão. Revista Brasileira de Engenharia Agrícola e Ambiental, v.3, p.349-353, 1999.

Almeida, D. P.; Resende, O.; Costa, L. M.; Mendes, U. C.; Sal, J. F. Cinética de secagem do feijão adzuki (Vigna angularis). Global Science Technology, v.2, p.72 - 83, 2009.

Amendola, M.; Queiroz, M. R. Mathematical methodologies for calculating the mass diffusion coefficient of bananas during drying. Revista Brasileira de Engenharia Agrícola e Ambiental, v.11, p.623-627, 2007.

Babalis, S. J.; Belessiotis, V. G. Influence of the drying conditions on the drying constants and moisture diffusivity during the thin-layer drying of figs. Journal of Food Engineering, v.65, p.449-58, 2004.

Brasil. Ministério da Agricultura e Reforma Agrária. Regras para análise de sementes. Brasília, DF. 2009.

Brooker, D. B., Bakker-arkema, F. W., Hall, C. W. Drying and storage of grains and oilseeds. Westport: The Avi Publishing Company, 1992. 450p. 
Doymaz, I. The kinetics of forced convective air-drying of pumpkin slices. Journal of Food Engineering, v.79, p.243249, 2007.

EMATER - Empresa de Assistência Técnica e Extensão Rural. Núcleo de Agronegócio: Produção e comercialização de pimenta no Distrito Federal - DF. 2006.

Goneli, A. L. D. Dinâmica da variação das propriedades físicomecânicas e da qualidade das sementes de mamona (Ricinus communis L.) durante a secagem e o armazenamento sob condições controladas. Viçosa: UFV, 2007. 192p. Tese Doutorado

Gravina, O.; Henz, G. P.; Carvalho, S. I. C. Conservação póscolheita de pimentas da espécie Capsicum chinense com filme PVC em duas temperaturas. In: Congresso Brasileiro de Olericultura, 44, 2004. Anais... Campo Grande: ABH, 2004. CD-Rom.

Kashani-Nejad, M. A.; Mortazavi, A.; Safekordi A. G. Thinlayer drying characteristics and modeling of pistachio nuts. Journal of Food Engineering, v.78, p. 98-108, 2007.

Lahsasni, S.; Kouhila, M.; Mahrouz, M.; Jaouhari, J. T. Drying kinetcs of prickly pear fruit (Opuntia ficus indica). Journal of Food Engineering, v.61, p.173-9, 2004.

Madamba, P. S.; Driscoll, R. H.; Buckle, K. A. Thin-layer drying characteristcs of garlic slices. Journal of Food Engineering, v.29, p.75-97, 1996.

Martinazzo, A. P.; Corrêa, P. C.; Melo, E. C.; Barbosa, F. F. Difusividade efetiva em folhas de Cymbopogon citratus (DC.) Stapf submetidas à secagem com diferentes comprimentos de corte e temperaturas do ar. Revista Brasileira de Plantas Medicinais, v.9, p.68-72, 2007a.
Martinazzo, A. P.; Corrêa, P. C.; Resende, O.; Melo, E. C.; Análise e descrição matemática da cinética de secagem de folhas de capim-limão. Revista Brasileira de Engenharia Agrícola e Ambiental, v.11, p.301-306, 2007b.

Midilli, A.; Kucuk, H.; Yapar, Z. A. New model for single-layer drying. Drying Technology, v.20, p.1503-1513, 2002.

Mohapatra, D.; Rao, P. S. A thin layer drying model of parboiled wheat. Journal of Food Engineering, v.66, p.513-18, 2005.

Oliveira, R. A.; Oliveira, W. P.; Park, K. J. Determinação da difusividade efetiva de raiz de chicória. Engenharia Agrícola, v.26, p.181-189, 2006.

Radünz, L. L.; Mossi, A. J.; Zakrzevski, C. A.; Amaral, A. S.; Grassmann, L. Análise da cinética de secagem de folhas de sálvia. Revista Brasileira de Engenharia Agrícola e Ambiental, v.14, p.979-986, 2010.

Resende, O.; Corrêa, P. C.; Goneli, A. L. D.; Botelho, F. M.; Rodrigues, S. Modelagem matemática do processo de secagem de duas variedades de feijão (Phaseolus vulgaris L.). Revista Brasileira de Produtos Agroindustriais, v.10, p.1726, 2008.

Rizvi, S. S. H. Thermodynamic properties of food in dehydration. In: Rao, M. A.; Rizvi, S. S. H. (eds.) Engineering properties of foods. New York: Marcel Dekker Inc, 1995. cap. 7. p.223-309.

Vilela, C. A. A.; Artur, P. O. Secagem do açafrão (Curcuma longa L.) em diferentes cortes geométricos. Ciência e Tecnologia de Alimentos, v.28, p. 387-394, 2008.

Zogzas, N. P.; Mauroulis, Z. B.; Marinos-Kouris, D. Moisture diffusivity data compilation in foodstuffs. Drying Technology, v.14, p.2225-2253, 1996. 